\title{
Aromatic cytokinin arabinosides promote PAMP-like responses and positively regulate leaf longevity
}

Magdaléna Bryksovái,\#, Siarhei Dabravolski2,\#, Zuzana Kučerová,\#, Filip Zavadil Kokáš ${ }^{2,4}$, Martina Špundová ${ }^{3}$, Lucie Plíhalová1,5, Tomáš Takáč ${ }^{6}$, Jiří Grúz ${ }^{5}$, Martin Hudeček ${ }^{5}$, Veronika Hloušková ${ }^{5}$, Radoslav Koprna ${ }^{1}$, Ondřej Novák ${ }^{5}$, Miroslav Strnad ${ }^{5}$, Ondřej Plíhal ${ }^{2,5, *}$, and Karel Doležal ${ }^{1,5}$

${ }^{1}$ Department of Chemical Biology and Genetics, Centre of the Region Haná for Biotechnological and Agricultural Research, Faculty of Science, Palacký University, Šlechtitelů 27, 78371 Olomouc, Czech Republic.

2 Department of Molecular Biology, Centre of the Region Haná for Biotechnological and Agricultural Research, Faculty of Science, Palacký University, Šlechtitelů 27, 78371 Olomouc, Czech Republic.

${ }^{3}$ Department of Biophysics, Centre of the Region Haná for Biotechnological and Agricultural Research, Faculty of Science, Palacký University, Šlechtitelů 27, 78371 Olomouc, Czech Republic.

${ }^{4}$ Regional Centre for Applied Molecular Oncology (RECAMO), Masaryk Memorial Cancer Institute, Žlutý kopec 7, 65653 Brno, Czech Republic.

${ }^{5}$ Laboratory of Growth Regulators, Faculty of Science, Institute of Experimental Botany of the Czech Academy of Sciences \& Palacký University, Šlechtitelů 27, 78371 Olomouc, Czech Republic.

${ }^{6}$ Department of Cell Biology, Centre of the Region Haná for Biotechnological and Agricultural Research, Faculty of Science, Palacký University, Šlechtitelů 27, 78371 Olomouc, Czech Republic.

* Corresponding Author: ondrej.plihal@upol.cz

\section{Supporting Information}

Supplementary Methods pp. 2-5

Supplementary Tables pp. 6-12

Supplementary References pp. 13 


\section{Supplementary Methods}

\section{Synthesis of prepared derivatives}

The synthesis of BAPAs was achieved according to the following general reaction scheme given here:

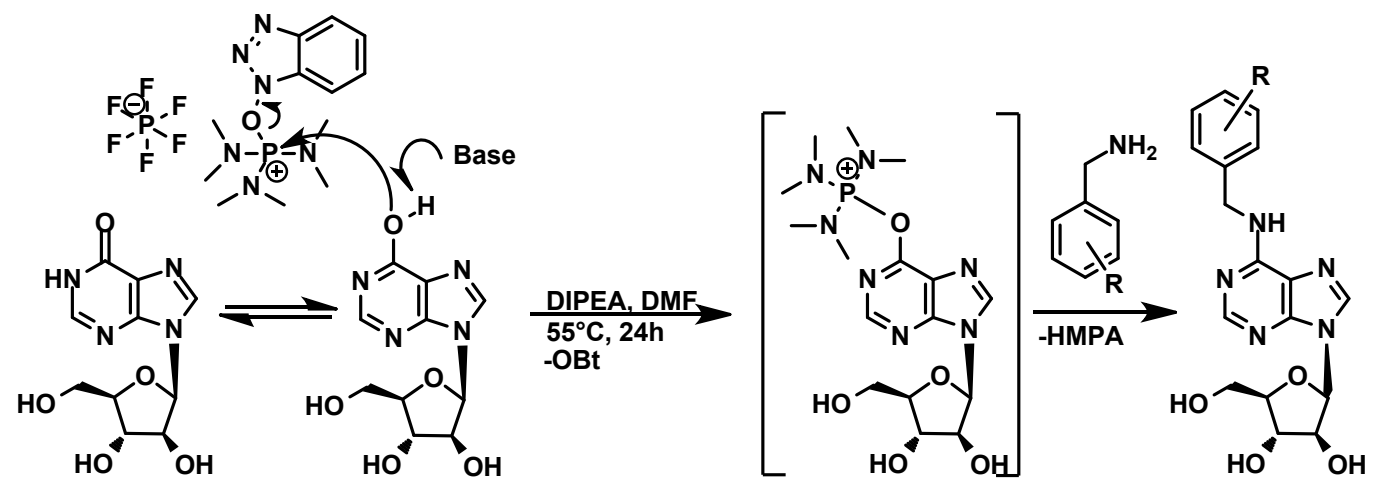

9-( $\beta$-D-arabinofuranosyl)hypoxantine $(100 \mathrm{mg}, 0.37 \mathrm{mmol})$ and BOP $(196 \mathrm{mg}, 0.37 \mathrm{mmol})$ were mixed together in DMF $(2 \mathrm{ml})$. Subsequently, the appropriate benzylamine $(0.37 \mathrm{mmol})$ and DIPEA $(97 \mu \mathrm{l}$, $0.44 \mathrm{mmol}$ ) were added. The reaction mixture was stirred under an argon atmosphere in an oil bath at $60{ }^{\circ} \mathrm{C}$ for $24 \mathrm{~h}$ then evaporated using a rotary evaporator. $\mathrm{MeOH}$ with a drop of chloroform was added to the distillation residue and the mixture was sonicated. The resulted white solid was isolated by filtration and re-crystallized from EtOH. The identity and purity of the synthesized compounds was confirmed by elemental and melting point analysis, analytical thin layer chromatography, high performance liquid chromatography, ES+MS spectrometry, and ${ }^{1} \mathrm{H}$ NMR (Supplementary Table 1).

${ }^{1} \mathrm{H}$ NMR data for the new compounds (DMSO-d6, $\left.500 \mathrm{MHz}\right) \delta \mathrm{ppm}$ :

Compound 1:

3.55-3.75 (m,3H), $4.09(\mathrm{t}, \mathrm{J}=4,5 \mathrm{~Hz}, 2 \mathrm{H}), 4.66(\mathrm{bs}, 2 \mathrm{H}), 5.05(\mathrm{t}, \mathrm{J}=5,5 \mathrm{~Hz}, 1 \mathrm{H}), 5.48(\mathrm{~d}, \mathrm{~J}=4,5 \mathrm{~Hz}, 1 \mathrm{H}), 5.57$ $(\mathrm{d}, \mathrm{J}=5 \mathrm{~Hz}, 1 \mathrm{H}), 6.21(\mathrm{~d}, \mathrm{~J}=4 \mathrm{~Hz}, 1 \mathrm{H}), 7.12-7.32(\mathrm{~m}, 5 \mathrm{H}), 8.14(\mathrm{~s}, 1 \mathrm{H}), 8.16(\mathrm{~s}, 1 \mathrm{H}), 8.32(\mathrm{bs}, 1 \mathrm{H})$

Compound 2:

3.55-3.68 (m,2H), $3.74(\mathrm{~d}, \mathrm{~J}=3,5 \mathrm{~Hz}, 1 \mathrm{H}), 4.10(\mathrm{~s}, 2 \mathrm{H}), 4.71(\mathrm{bs}, 2 \mathrm{H}), 5.05(\mathrm{~d}, \mathrm{~J}=4,5 \mathrm{~Hz}, 1 \mathrm{H}), 5.49(\mathrm{~s}, 1 \mathrm{H})$, $5.58(\mathrm{~d}, \mathrm{~J}=3,5 \mathrm{~Hz}, 1 \mathrm{H}), 6.23(\mathrm{~d}, \mathrm{~J}=3,5 \mathrm{~Hz}, 1 \mathrm{H}), 7.03-7.32(\mathrm{~m}, 4 \mathrm{H}), 8.15(\mathrm{~s}, 1 \mathrm{H}), 8.18(\mathrm{~s}, 1 \mathrm{H}), 8.30(\mathrm{bs}, 1 \mathrm{H})$

Compound 3:

3.55-3.67 (m,2H), $3.74 \quad(\mathrm{~d}, \mathrm{~J}=4 \mathrm{~Hz}, 1 \mathrm{H}), 4.10 \quad(\mathrm{~s}, 2 \mathrm{H}), \quad 4.67 \quad(\mathrm{bs}, 2 \mathrm{H}), \quad 5.05 \quad(\mathrm{t}, \mathrm{J}=4,5 \mathrm{~Hz}, 1 \mathrm{H}), \quad 5.48$ $(\mathrm{d}, \mathrm{J}=4 \mathrm{~Hz}, 1 \mathrm{H}), 5.57(\mathrm{~d}, \mathrm{~J}=3,5 \mathrm{~Hz}, 1 \mathrm{H}), 6.22(\mathrm{~d}, \mathrm{~J}=4 \mathrm{~Hz}, 1 \mathrm{H}), 6.98(\mathrm{t}, \mathrm{J}=6 \mathrm{~Hz}, 1 \mathrm{H}), 7.06-7.16(\mathrm{~m}, 2 \mathrm{H}), 7.25-$ $7.33(\mathrm{~m}, 1 \mathrm{H}), 8.15(\mathrm{~s}, 1 \mathrm{H}), 8.18(\mathrm{~s}, 1 \mathrm{H}), 8.37(\mathrm{bs}, 1 \mathrm{H})$

Compound 4:

3.55-3.66 (m,2H), $3.73(\mathrm{~d}, \mathrm{~J}=4,5 \mathrm{~Hz}, 1 \mathrm{H}), 4.07-4.11(\mathrm{~m}, 2 \mathrm{H}), 4.62(\mathrm{bs}, 2 \mathrm{H}), 5.06(\mathrm{t}, \mathrm{J}=5,5 \mathrm{~Hz}, 1 \mathrm{H}), 5.49$ $(\mathrm{d}, \mathrm{J}=4,5 \mathrm{~Hz}, 1 \mathrm{H}), 5.57(\mathrm{~d}, \mathrm{~J}=5 \mathrm{Jz}, 1 \mathrm{H}), 6.22(\mathrm{~d}, \mathrm{~J}=5 \mathrm{~Hz}, 1 \mathrm{H}), 7.07(\mathrm{t}, \mathrm{J}=8,5 \mathrm{~Hz}, 2 \mathrm{H}), 7.33(\mathrm{t}, \mathrm{J}=5,5 \mathrm{~Hz}, 2 \mathrm{H})$, $8.16(\mathrm{~s}, 2 \mathrm{H}), 8.34(\mathrm{bs}, 1 \mathrm{H})$

Compound 5: 
3.54-3.80 (m,3H), $4.10(\mathrm{bs}, 2 \mathrm{H}), 4.71(\mathrm{bs}, 2 \mathrm{H}), 5.06(\mathrm{bs}, 1 \mathrm{H}), 5.49(\mathrm{~d}, \mathrm{~J}=3,5 \mathrm{~Hz}, 1 \mathrm{H}), 5.60(\mathrm{~d}, \mathrm{~J}=4 \mathrm{~Hz}, 1 \mathrm{H})$, $6.23(\mathrm{~d}, \mathrm{~J}=4 \mathrm{~Hz}, 1 \mathrm{H}) 7.21(\mathrm{~d}, \mathrm{~J}=4 \mathrm{~Hz}, 3 \mathrm{H}), 7.37-7.44(\mathrm{~m}, 1 \mathrm{H}), 8.13(\mathrm{~s}, 1 \mathrm{H}), 8.20(\mathrm{~s}, 1 \mathrm{H}), 8.34(\mathrm{bs}, 1 \mathrm{H})$

Compound 6:

3.54-3.65 (m,2H), $3.74(\mathrm{bs}, 1 \mathrm{H}), 4.09(\mathrm{bs}, 2 \mathrm{H}), 4.65(\mathrm{bs}, 2 \mathrm{H}), 5.11(\mathrm{bs}, 1 \mathrm{H}), 5.56(\mathrm{bs}, 2 \mathrm{H}), 6.21$ (d, J=4,5 $\mathrm{Hz}, 1 \mathrm{H}), 7.21-7.35(\mathrm{~m}, 4 \mathrm{H}), 8.15(\mathrm{~s}, 1 \mathrm{H}), 8.17(\mathrm{~s}, 1 \mathrm{H}), 8.37(\mathrm{~s}, 1 \mathrm{H})$

Compound 7:

3.56-3.67 (m,2H), $3.73(\mathrm{bs}, 1 \mathrm{H}), 4.09(\mathrm{bs}, 2 \mathrm{H}), 4.63(\mathrm{bs}, 2 \mathrm{H}), 5.05(\mathrm{t}, \mathrm{J}=4,5 \mathrm{~Hz}, 1 \mathrm{H}), 5.48(\mathrm{~d}, \mathrm{~J}=3 \mathrm{~Hz}, 1 \mathrm{H})$, $5.57(\mathrm{~d}, \mathrm{~J}=4,5 \mathrm{~Hz}, 1 \mathrm{H}), 6.22(\mathrm{~d}, \mathrm{~J}=4 \mathrm{~Hz}, 1 \mathrm{H}), 7.30(\mathrm{~s}, 4 \mathrm{H}), 8.14(\mathrm{~s}, 1 \mathrm{H}), 8.17(\mathrm{~s}, 1 \mathrm{H}), 8.36(\mathrm{bs}, 1 \mathrm{H})$

\section{Compound 8:}

3.75-3.79 (m,2H), $4.13(\mathrm{t}, \mathrm{J}=5 \mathrm{~Hz}, 3 \mathrm{H}), 4.64(\mathrm{bs}, 2 \mathrm{H}), 5.23(\mathrm{bs}, 1 \mathrm{H}), 5.66(\mathrm{bs}, 2 \mathrm{H}), 6.25(\mathrm{~d}, \mathrm{~J}=5,5 \mathrm{~Hz}, 1 \mathrm{H})$, $7.09(\mathrm{t}, \mathrm{J}=7,5 \mathrm{~Hz}, 1 \mathrm{H}), 7.33(\mathrm{~d}, \mathrm{~J}=7,5 \mathrm{~Hz}, 1 \mathrm{H}), 7.55(\mathrm{~d}, \mathrm{~J}=8 \mathrm{~Hz}, 1 \mathrm{H}), 7.68(\mathrm{~s}, 1 \mathrm{H}), 8.18(\mathrm{~s}, 1 \mathrm{H}), 8.21(\mathrm{~s}, 1 \mathrm{H})$, $8.36(\mathrm{bs}, 1 \mathrm{H})$

\section{Compound 9:}

3.54-3.65 (m,2H), $3.72(\mathrm{~d}, \mathrm{~J}=4 \mathrm{~Hz}, 1 \mathrm{H}), 4.09(\mathrm{t}, \mathrm{J}=4,5 \mathrm{~Hz}, 2 \mathrm{H}), 4.58(\mathrm{bs}, 2 \mathrm{H}), 5.04(\mathrm{t}, \mathrm{J}=5 \mathrm{~Hz}, 1 \mathrm{H}), 5.48$ $(\mathrm{d}, \mathrm{J}=4 \mathrm{~Hz}, 1 \mathrm{H}), 5.56(\mathrm{~d}, \mathrm{~J}=4,5 \mathrm{~Hz}, 1 \mathrm{H}), 6.21(\mathrm{~d}, \mathrm{~J}=4 \mathrm{~Hz}, 1 \mathrm{H}), 7.09(\mathrm{~d}, \mathrm{~J}=8,5 \mathrm{~Hz}, 2 \mathrm{H}), 7.59(\mathrm{~d}, \mathrm{~J}=8 \mathrm{~Hz}, 2 \mathrm{H}), 8.13$ $(\mathrm{s}, 1 \mathrm{H}), 8.16(\mathrm{~s}, 1 \mathrm{H}), 8.34(\mathrm{bs}, 1 \mathrm{H})$

Compound 10:

3.55-3.68 (m,2H), $3.74(\mathrm{~d}, \mathrm{~J}=4 \mathrm{~Hz}, 1 \mathrm{H}), 3.79(\mathrm{~s}, 3 \mathrm{H}), 4.10(\mathrm{~s}, 2 \mathrm{H}), 4.62(\mathrm{bs}, 2 \mathrm{H}), 5.07(\mathrm{bs}, 1 \mathrm{H}), 5.50(\mathrm{~s}, 1 \mathrm{H})$, $5.59(\mathrm{~s}, 1 \mathrm{H}), 6.23(\mathrm{~d}, \mathrm{~J}=4 \mathrm{~Hz}, 1 \mathrm{H}), 6.79(\mathrm{t}, \mathrm{J}=8 \mathrm{~Hz}, 1 \mathrm{H}), 6.93(\mathrm{~d}, \mathrm{~J}=7,5 \mathrm{~Hz}, 1 \mathrm{H}), 7.05(\mathrm{~d}, \mathrm{~J}=6,5 \mathrm{~Hz}, 1 \mathrm{H}), 7.16$ $(\mathrm{t}, \mathrm{J}=8 \mathrm{~Hz}, 1 \mathrm{H}), 8.06(\mathrm{~s}, 1 \mathrm{H}), 8.11(\mathrm{~s}, 1 \mathrm{H}), 8.17(\mathrm{bs}, 1 \mathrm{H})$

Compound 11:

3.56-3.79 (m,6H), $4.10(\mathrm{~s}, 2 \mathrm{H}), 4.63(\mathrm{bs}, 2 \mathrm{H}), 5.06(\mathrm{~s}, 1 \mathrm{H}), 5.49(\mathrm{~s}, 1 \mathrm{H}), 5.58(\mathrm{~s}, 1 \mathrm{H}), 6.22(\mathrm{~d}, \mathrm{~J}=2,5 \mathrm{~Hz}, 1 \mathrm{H})$, $6.72(\mathrm{~d}, \mathrm{~J}=6 \mathrm{~Hz}, 1 \mathrm{H}), 6.86(\mathrm{~d}, \mathrm{~J}=8 \mathrm{~Hz}, 2 \mathrm{H}), 7.15(\mathrm{t}, \mathrm{J}=8 \mathrm{~Hz}, 1 \mathrm{H}), 8.15(\mathrm{~s}, 1 \mathrm{H}), 8.17(\mathrm{~s}, 1 \mathrm{H}), 8.30(\mathrm{bs}, 1 \mathrm{H})$

\section{Compound 12:}

3.52-3.75 (m,6H), 4.03-4.12 (m,2H), $4.56(\mathrm{bs}, 2 \mathrm{H}), 5.00-5.09(\mathrm{~m}, 1 \mathrm{H}), 5.44-5.59(\mathrm{~m}, 2 \mathrm{H}), 6.15-6.24$ $(\mathrm{m}, 1 \mathrm{H}), 6.79(\mathrm{~d}, \mathrm{~J}=8,5 \mathrm{~Hz}, 2 \mathrm{H}), 7.16-7.26(\mathrm{~m}, 2 \mathrm{H}), 8.12(\mathrm{~s}, 1 \mathrm{H}), 8.14(\mathrm{~s}, 1 \mathrm{H}), 8.24(\mathrm{bs}, 1 \mathrm{H})$

\section{Compound 13:}

3.54-3.67 (m,2H), $3.74(\mathrm{~d}, \mathrm{~J}=3,5 \mathrm{~Hz}, 1 \mathrm{H}), 4.10(\mathrm{~s}, 2 \mathrm{H}), 4.57(\mathrm{bs}, 2 \mathrm{H}), 5.06(\mathrm{~s}, 1 \mathrm{H}), 5.49(\mathrm{~s}, 1 \mathrm{H}), 5.58$ $(\mathrm{d}, \mathrm{J}=3,5 \mathrm{~Hz}, 1 \mathrm{H}), 6.22(\mathrm{~d}, \mathrm{~J}=3 \mathrm{~Hz}, 1 \mathrm{H}), 6.54(\mathrm{~d}, \mathrm{~J}=7,5 \mathrm{~Hz}, 1 \mathrm{H}), 6.65-6.74(\mathrm{~m}, 2 \mathrm{H}), 7.02(\mathrm{t}, \mathrm{J}=7,5 \mathrm{~Hz}, 1 \mathrm{H}), 8.14$ $(\mathrm{s}, 1 \mathrm{H}), 8.16(\mathrm{~s}, 1 \mathrm{H}), 8.26(\mathrm{bs}, 1 \mathrm{H}), 9.20(\mathrm{~s}, 1 \mathrm{H})$

\section{Compound 14:}

3.54-3.76 (m,6H), 4.03-4.15 (m,2H), $4.62(\mathrm{bs}, 1 \mathrm{H}), 5.04(\mathrm{t}, \mathrm{J}=5,5 \mathrm{~Hz}, 1 \mathrm{H}), 5.49(\mathrm{~d}, \mathrm{~J}=4,5 \mathrm{~Hz}, 2 \mathrm{H}), 5.59$ $(\mathrm{d}, \mathrm{J}=6 \mathrm{~Hz}, 2 \mathrm{H}), 6.12-6.25(\mathrm{~m}, 1 \mathrm{H}), 7.01-7.18(\mathrm{~m}, 2 \mathrm{H}), 7.99(\mathrm{~s}, 1 \mathrm{H}), 8.10(\mathrm{~s}, 1 \mathrm{H}), 8.12(\mathrm{~s}, 1 \mathrm{H}), 8.23(\mathrm{bs}, 1 \mathrm{H})$

\section{Compound 15:}

3.55-3.78 (m,6H), 4.06-4.15 (m,2H), $4.62(\mathrm{bs}, 2 \mathrm{H}), 5.14(\mathrm{t}, \mathrm{J}=5,5 \mathrm{HZ}, 1 \mathrm{H}), 5.56(\mathrm{~d}, \mathrm{~J}=4 \mathrm{~Hz}, 1 \mathrm{H}), 5.62$ $(\mathrm{d}, \mathrm{J}=5 \mathrm{~Hz}, 1 \mathrm{H}), 6.22(\mathrm{~d}, \mathrm{~J}=4 \mathrm{~Hz}, 1 \mathrm{H}), 6.96(\mathrm{~d}, \mathrm{~J}=7 \mathrm{~Hz}, 1 \mathrm{H}), 7.04-7.15(\mathrm{~m}, 3 \mathrm{H}), 8.14(\mathrm{~s}, 1 \mathrm{H}), 8.16(\mathrm{~s}, 1 \mathrm{H}), 8.26$ (bs, $1 \mathrm{H})$

\section{Compound 16:}

3.54-3.77 (m,6H), $4.09(\mathrm{~s}, 2 \mathrm{H}), \quad 4.61 \quad(\mathrm{bs}, 2 \mathrm{H}), \quad 5.05(\mathrm{t}, \mathrm{J}=5,5 \mathrm{~Hz}, 1 \mathrm{H}), \quad 5.48 \quad(\mathrm{~d}, \mathrm{~J}=3,5 \mathrm{~Hz}, 1 \mathrm{H}), \quad 5.57$ $(\mathrm{d}, \mathrm{J}=4,5 \mathrm{~Hz}, 1 \mathrm{H}), 6.21(\mathrm{~d}, \mathrm{~J}=4 \mathrm{~Hz}, 1 \mathrm{H}), 7.04(\mathrm{~d}, \mathrm{~J}=7,5 \mathrm{~Hz}, 2 \mathrm{H}), 7.17(\mathrm{~d}, \mathrm{~J}=7,5 \mathrm{~Hz}, 2 \mathrm{H}), 8.13(\mathrm{~s}, 1 \mathrm{H}), 8.15(\mathrm{~s}, 1 \mathrm{H})$, $8.26(\mathrm{bs}, 1 \mathrm{H})$ 
Compound 17:

3.57-3.70 (m,2H), $3.76(\mathrm{~d}, \mathrm{~J}=4 \mathrm{~Hz}, 1 \mathrm{H}), 4.12(\mathrm{~s}, 2 \mathrm{H}), 4.51(\mathrm{bs}, 2 \mathrm{H}), 5.09(\mathrm{t}, \mathrm{J}=5 \mathrm{~Hz}, 1 \mathrm{H}), 5.19(\mathrm{~s}, 2 \mathrm{H}), 5.52$

$(\mathrm{d}, \mathrm{J}=4 \mathrm{~Hz}, 1 \mathrm{H}), 5.59(\mathrm{~d}, \mathrm{~J}=5 \mathrm{~Hz}, 1 \mathrm{H}), 6.25(\mathrm{~d}, \mathrm{~J}=4 \mathrm{~Hz}, 1 \mathrm{H}), 6.45(\mathrm{t}, \mathrm{J}=7 \mathrm{~Hz}, 1 \mathrm{H}), 6.59(\mathrm{~d}, \mathrm{~J}=8 \mathrm{~Hz}, 1 \mathrm{H}), 6.90$

$(\mathrm{t}, \mathrm{J}=7 \mathrm{~Hz}, 1 \mathrm{H}), 7.07(\mathrm{~d}, \mathrm{~J}=7,5 \mathrm{~Hz}, 1 \mathrm{H}), 8.20(\mathrm{~s}, 3 \mathrm{H})$

Compound 18:

3.55-3.76 (m,3H), 4.06-4.13 (m,2H), $4.73(\mathrm{bs}, 2 \mathrm{H}), 5.05 \quad(\mathrm{t}, \mathrm{J}=5 \mathrm{~Hz}, 1 \mathrm{H}), \quad 5.48 \quad(\mathrm{~d}, \mathrm{~J}=4,5 \mathrm{~Hz}, 1 \mathrm{H})$, $5.56(\mathrm{~d}, \mathrm{~J}=5,5 \mathrm{~Hz}, 1 \mathrm{H}), 6.22(\mathrm{~d}, \mathrm{~J}=5 \mathrm{~Hz}, 1 \mathrm{H}), 7.46-7.68(\mathrm{~m}, 4 \mathrm{H}), 8.15(\mathrm{~s}, 1 \mathrm{H}), 8.18(\mathrm{~s}, 1 \mathrm{H}), 8.44(\mathrm{bs}, 1 \mathrm{H})$

Compound 19:

3.56-3.76 (m,3H), 4.05-4.14 (m,2H), $4.68(\mathrm{bs}, 2 \mathrm{H}), 5.05(\mathrm{t}, \mathrm{J}=5,5 \mathrm{~Hz}, 1 \mathrm{H}), 5.48(\mathrm{~d}, \mathrm{~J}=4,5 \mathrm{~Hz}, 1 \mathrm{H}), 5.57$ $(\mathrm{d}, \mathrm{J}=5,5 \mathrm{~Hz}, 1 \mathrm{H}), 6.22(\mathrm{~d}, \mathrm{~J}=4,5 \mathrm{~Hz}, 1 \mathrm{H}), 7.15(\mathrm{~d}, \mathrm{~J}=8 \mathrm{~Hz}, 1 \mathrm{H}), 7.25-7.42(\mathrm{~m}, 3 \mathrm{H}), 8.15(\mathrm{~s}, 1 \mathrm{H}), 8.18(\mathrm{~s}, 1 \mathrm{H})$, 8.40 (bs, $1 \mathrm{H})$

Compound 20:

3.54-3.76 (m,3H), 4.03-4.15 (m,2H), $4.68(\mathrm{bs}, 2 \mathrm{H}), 5.05(\mathrm{t}, \mathrm{J}=5,5 \mathrm{~Hz}, 1 \mathrm{H}), 5.48(\mathrm{~d}, \mathrm{~J}=4 \mathrm{~Hz}, 1 \mathrm{H}), 5.56$

$(\mathrm{d}, \mathrm{J}=5 \mathrm{~Hz}, 1 \mathrm{H}), 6.23(\mathrm{~d}, \mathrm{~J}=4,5 \mathrm{~Hz}, 1 \mathrm{H}), 7.02-7.22(\mathrm{~m}, 3 \mathrm{H}), 8.16(\mathrm{~s}, 1 \mathrm{H}), 8.19(\mathrm{~s}, 1 \mathrm{H}), 8.34(\mathrm{bs}, 1 \mathrm{H})$

Compound 21:

3.40-3.53 (m,2H), $3.60(\mathrm{~d}, \mathrm{~J}=2,5 \mathrm{~Hz}, 1 \mathrm{H}), 3.95(\mathrm{~s}, 2 \mathrm{H}), 4.52(\mathrm{bs}, 2 \mathrm{H}), 4.91 \mathrm{t}, \mathrm{J}=5 \mathrm{~Hz}, 1 \mathrm{~h}), 5.35(\mathrm{~d}, \mathrm{~J}=2 \mathrm{~Hz}, 1 \mathrm{H})$, $5.42(\mathrm{~d}, \mathrm{~J}=4 \mathrm{~Hz}, 1 \mathrm{H}), 6.09(\mathrm{~d}, \mathrm{~J}=3 \mathrm{~Hz}, 1 \mathrm{H}), 6.79-6.92(\mathrm{~m}, 3 \mathrm{H}), 8.02(\mathrm{~s}, 1 \mathrm{H}), 8.05(\mathrm{~s}, 1 \mathrm{H}), 8.26(\mathrm{bs}, 1 \mathrm{H})$

\section{General procedures}

Elemental analyses were performed using an EA1112 CHN analyzer (Thermo Finnigan, Thermofisher Scientific). Melting points were determined on a Buchi Melting Point B-540 apparatus and are uncorrected. Analytical thin-layer chromatography (TLC) was carried out using silica gel $60 \mathrm{WF}_{254}$ plates (Merck) with $\mathrm{CHCl}_{3} / \mathrm{MeOH}(4: 1, \mathrm{v} / \mathrm{v})$ as the solvent. The purity of the synthesized compounds was determined as described previously ${ }^{l}$. Briefly, samples were dissolved in methanol $\left(1 \mathrm{mg} \mathrm{ml}^{-1}\right)$ and then diluted to a concentration of $10 \mu \mathrm{g} \mathrm{m}^{-1}$ in the initial mobile phase. $10 \mu \mathrm{l}$ of the resulting solution was injected onto a thermostated $\left(25^{\circ} \mathrm{C}\right) \mathrm{RP}$ column $(150 \mathrm{~mm} \times 2.1 \mathrm{~mm}, 5 \mu \mathrm{m}$ C18 Symmetry, Waters) and analyzed using an Alliance 2695 Separations Module high-performance liquid chromatograph coupled to a PDA 2996 detector (Waters) with detection at wavelengths of 210-400 nm. The chromatograph's effluent was directed into the ion source of a benchtop quadrupole orthogonal acceleration time-of-flight Q-TOF Micro tandem mass spectrometer (Waters). HPLC elution was performed at a flow rate of $0.3 \mathrm{ml} \mathrm{min}{ }^{-1}$ using a linear gradient of $15 \mathrm{mM}$ ammonium formate at $\mathrm{pH}$ 4.0 (mobile phase A) and pure methanol (mobile phase B). The proportion of B in the mobile phase was initially $10 \%$ and was increased linearly to $90 \%$ over $24 \mathrm{~min}$, after which isocratic elution was performed with $90 \% \mathrm{~B}$. The column was then re-equilibrated under the initial conditions $(10 \% \mathrm{~B})$ for $10 \mathrm{~min}$. The electrospray source was maintained at $120^{\circ} \mathrm{C}$ with a capillary voltage of $+3.0 \mathrm{kV}$, a cone voltage of $+20 \mathrm{~V}$, and a desolvation temperature of $300^{\circ} \mathrm{C}$. Nitrogen was used as both the desolvation gas (500 1 $\left.\mathrm{h}^{-1}\right)$ and the cone gas $\left(50 \mathrm{l} \mathrm{h}^{-1}\right)$. The mass spectrometer was operated in positive (ESI+) ionization mode and data were acquired in the $50-1000 \mathrm{~m} / \mathrm{z}$ range. The mass spectrometer was directly coupled to a MassLynx 4.1 data system. ${ }^{1} \mathrm{H}$ NMR spectra were measured on a JEOL 500 ECA instrument at $500 \mathrm{MHz}$. Samples were prepared by dissolving the compounds in DMSO- $d_{6}$. Tetramethylsilane (TMS) was used as the internal standard. 


\section{KEGG pathway over-representation analysis}

For the visual interpretation of transcriptomic data from 3-methoxy-BAPA treatments, the KEGG database was employed (https://www.kegg.jp). KEGG Mapper was used for processing of KO annotation data ${ }^{2}$.

\section{Bacterial cytokinin assay}

E. coli strain KMI001 ( $4 r c s C$, cps::lacZ), harboring either the plasmid pIN-III-AHK4 or pSTV28AHK3, which express the Arabidopsis histidine kinases CRE1/AHK4 or AHK3 ${ }^{3,4}$ was used in the experiments. Activity of $\beta$-galactosidase was measured using 4-methylumbelliferyl $\beta$-Dgalactopyranoside as a substrate after overnight growth in the presence of $\mathrm{CK}^{5}$. In addition, live-cell cytokinin-binding assay with radiolabeled $t \mathrm{Z}$ as a competitor was performed as previously described ${ }^{6}$. Homogenous bacterial suspensions with $\mathrm{OD}_{600}$ values of 0.8 and 1.2 were found to be optimal for CRE1/AHK4 and AHK3 cultures, respectively. The competition reaction was allowed to proceed with $2 \mathrm{nM}\left[2-{ }^{3} \mathrm{H}\right] t \mathrm{Z}$ and various concentrations of the tested compounds for $30 \mathrm{~min}$ at $4{ }^{\circ} \mathrm{C}$. When binding equilibrium was reached, the suspension was centrifuged $(6000 \mathrm{xg})$, the supernatant was removed, and the bacterial pellet was resuspended in the scintillation cocktail (Beckman).

\section{Effect of 3-methoxy-BAPA and 3-hydroxy-BAPA on infection level in field trial experiments}

An effect of 3-methoxy-BAPA and 3-hydroxy-BAPA was tested in field trials on winter wheat (cv. Turandot; Selgen, Czech Republic) and spring barley (cv. Francin; Selgen, Czech Republic) performed near Olomouc, Czech Republic (GPS: 49.5748542N, 17.2851261E; altitude $218 \mathrm{~m}$ ) in a growth season 2017/2018. The $5 \mu \mathrm{M}$ solution of 3-methoxy-BAPA or 3-hydroxy-BAPA was applied as a foliar spray on wheat at a growth stage of tillering (BBCH $25^{7}$ ) and on barley at stage of beginning of stem elongation (BBCH 30-33 ${ }^{7}$ ) or start of flag leaf extending (BBCH 39-41 ${ }^{7}$ ) in amount of $300 \mathrm{ml}$ per 10 $\mathrm{m}^{2}$. A level of infection was evaluated at a growth stage of beginning of heading $\left(\mathrm{BBCH} 51^{7}\right.$ ) in the case of wheat and leaf rusts and barley and powdery mildew or at growth stage of early milk ripeness $\left(\mathrm{BBCH} 73^{7}\right.$ ) in the case of barley and foot rot diseases. The evaluation of each variant was carried out on 5 plots $(\mathrm{n}=5), 20$ plants were evaluated from each plot. The level of infection was evaluated according the following scales:

i) for wheat and leaf rusts (namely Puccinia striiformis syn. Puccinia glumarum, Puccinia recondita f. sp. tritici, Puccinia graminis): 1 - without infection; 2 - a few clusters of chlorosis; 3 clusters to $1 \%$ of leaf area; 4 - clusters from 1 to $3 \%$ of leaf area; 5 - clusters from 3 to $5 \%$ of leaf area, beginning of strips; 6 - clusters and strips from 5 to $15 \%$ of leaf area;

ii) for barley and powdery mildew (Blumeria graminis syn. Erysiphe graminis): 1 - without symptoms; 2 - mycelium at max. $1 \%$ of leaf area; 3 -mycelium at $1-5 \%$ of leaf area; 4 -mycelium at $5-10 \%$ of leaf area; 5 - mycelium at $10-30 \%$ of leaf area;

iii) for barley and foot rot diseases (Gaeumannomyces graminis syn. Ophiobolus graminis, Ramulispora herpotrichoides syn. Pseudocercosporella herpotrichoides, teleomorpha Tapesia yallundae, Fusarium spp., Rhizoctonia spp.): 1 - without symptoms; 3 - brown spots to $5 \%$ of stem perimeter; 5 - brown spots from 5 to $25 \%$ of stem perimeter.

In the end of each experiment, a number of tillers per plant was determined with 50 randomized plants of the variant (10 plants from each plot). A number of spikes per $0.25 \mathrm{~m}^{2}$ was counted and converted to $1 \mathrm{~m}^{2}$. After harvest, grain yield was determined. 


\section{Supplementary Tables}

Supplementary Table 1. Elemental analysis, ES+ mass spectrometry analysis, melting points and HPLC purity of prepared BAPA derivatives.

\begin{tabular}{|c|c|c|c|c|c|c|}
\hline \multirow[t]{2}{*}{ Compound } & \multicolumn{3}{|c|}{ Elemental analysis calculated/found } & \multirow[t]{2}{*}{ Mp $\left({ }^{\circ} \mathrm{C}\right)$} & \multirow{2}{*}{$\begin{array}{l}\text { ES-MS } \\
{\left[\mathrm{M}+\mathrm{H}^{+}\right]}\end{array}$} & \multirow[t]{2}{*}{ HPLC (\%) } \\
\hline & $\%$ of $\mathrm{C}$ & $\%$ of $\mathrm{H}$ & $\%$ of $\mathrm{N}$ & & & \\
\hline 1 & $57.1 / 55.9$ & $5.3 / 4.9$ & $19.6 / 19.0$ & $191-193$ & 358 & $>98$ \\
\hline 2 & $54.4 / 52.6$ & $4.8 / 4.7$ & $18.7 / 18.0$ & $191-193$ & 376 & $>98$ \\
\hline 3 & $54.4 / 54.0$ & $4.8 / 4.7$ & $18.7 / 18.8$ & $224-225$ & 376 & $>98$ \\
\hline 4 & $54.4 / 52.9$ & $4.8 / 4.9$ & $18.7 / 18.0$ & $190-192$ & 376 & $>98$ \\
\hline 5 & $52.1 / 51.7$ & $4.6 / 4.7$ & $17.9 / 17.6$ & $226-229$ & 393 & $>99$ \\
\hline 6 & $52.1 / 51.9$ & $4.6 / 4.6$ & $17.9 / 17.4$ & $240-242$ & 393 & $>98$ \\
\hline 7 & $52.1 / 52.3$ & $4.6 / 4.4$ & $17.9 / 17.2$ & $181-183$ & 393 & $>99$ \\
\hline 8 & $42.3 / 41.8$ & $3.8 / 3.6$ & $14.5 / 14.1$ & $221-223$ & 484 & $>98$ \\
\hline 9 & $42.3 / 42.2$ & $3.8 / 3.8$ & $14.5 / 13.8$ & $182-184$ & 484 & $>98$ \\
\hline 10 & $55.8 / 55.6$ & $5.5 / 5.5$ & $18.1 / 17.9$ & $224-225$ & 388 & $>98$ \\
\hline 11 & $55.8 / 55.4$ & $5.5 / 5.4$ & $18.1 / 17.9$ & $236-238$ & 388 & $>99$ \\
\hline 12 & $55.8 / 55.7$ & $5.5 / 5.5$ & $18.1 / 17.9$ & $182-183$ & 388 & $>99$ \\
\hline 13 & $54.7 / 53.9$ & $5.1 / 5.0$ & $18.8 / 18.3$ & $235-238$ & 374 & $>98$ \\
\hline 14 & $58.2 / 57.9$ & $5.7 / 5.4$ & $18.9 / 18.8$ & $224-227$ & 372 & $>98$ \\
\hline 15 & $58.2 / 57.4$ & $5.7 / 5.3$ & $18.9 / 18.9$ & $216-218$ & 372 & $>99$ \\
\hline 16 & $58.2 / 58.0$ & $5.7 / 5.4$ & $18.9 / 18.6$ & $173-177$ & 372 & $>98$ \\
\hline 17 & $54.8 / 54.2$ & $5.4 / 5.3$ & $22.6 / 21.8$ & $193-196$ & 373 & $>98$ \\
\hline 18 & $50.8 / 49.9$ & $4.3 / 3.9$ & $16.5 / 15.8$ & $182-184$ & 426 & $>98$ \\
\hline 19 & $49.0 / 48.6$ & $4.1 / 3.9$ & $15.9 / 15.4$ & $175-176$ & 442 & $>98$ \\
\hline 20 & $51.9 / 51.2$ & $4.4 / 4.3$ & $17.8 / 17.6$ & $214-216$ & 394 & $>98$ \\
\hline 21 & $51.9 / 51.4$ & $4.4 / 3.7$ & $17.8 / 17.3$ & $236-237$ & 394 & $>98$ \\
\hline
\end{tabular}

Supplementary Table 2. Overview of DEGs regulated by 3-methoxy-BAPA. The table lists the 50 most strongly upregulated and downregulated genes (adjusted P-value $<0.05$ ), respectively, that showed the highest absolute $\log _{2}$ fold change $\left(\log _{2}\right.$ FC) after 6 h dark-incubation of detached Arabidopsis leaves with $10 \mu \mathrm{M} 3$-methoxy-BAPA.

\begin{tabular}{|c|c|c|}
\hline \multicolumn{3}{|c|}{ UPREGULATED DEGs } \\
\hline AGI number & Description & $\log _{2} \mathrm{FC}$ \\
\hline At2g47040 & Vanguard 1/Pectinesterase 5 [Source:Uniprot/SWISSPROT 3BAcc:Q5MFV8] & 2.80 \\
\hline At3g01270 & Probable pectate lyase 7 [Source:Uniprot/SWISSPROT 3BAcc:Q9SRH4] & 2.79 \\
\hline At $2 g 47050$ & $\begin{array}{l}\text { Plant invertase/pectin methylesterase inhibitor superfamily protein [Source:TAIR } \\
\text { 3BAcc:47050] }\end{array}$ & IR 2.31 \\
\hline At3g07820 & Pectin lyase-like superfamily protein [Source:TAIR 3BAcc:07820] & 2.22 \\
\hline At3g28750 & Hypothetical protein & 2.21 \\
\hline At2g47030 & $\begin{array}{l}\text { Plant invertase/pectin methylesterase inhibitor superfamily protein (VGDH1) } \\
\text { [Source:Uniprot/SWISSPROT 3BAcc:O80722] }\end{array}$ & 1) 2.16 \\
\hline At4g35010 & Beta-galactosidase 11 (BGAL11) [Source:Uniprot/SWISSPROT 3BAcc:Q9SCV1] & 2.03 \\
\hline At3g62230 & $\begin{array}{l}\text { F-box protein At3g62230/DUO1-ACTIVATED F-BOX } 1 \text { [Source:Uniprot/SWISSPROT } \\
\text { 3BAcc:Q9M1Q1] }\end{array}$ & T 1.95 \\
\hline At3g05610 & $\begin{array}{l}\text { Probable pectinesterase/pectinesterase inhibitor } 21 \text { [Source:Uniprot/SWISSPROT } \\
\text { 3BAcc:Q8GX86] }\end{array}$ & T 1.80 \\
\hline At $5 \mathrm{~g} 44430$ & $\begin{array}{l}\text { PLANT DEFENSIN } 1.2 \mathrm{C} \quad(\text { PDF1.2C)/Defensin-like } \\
\text { [Source:Uniprot/SWISSPROT 3BAcc:Q9FI22] }\end{array}$ & 171.75 \\
\hline Atlg55560 & SKU5 similar 14/SKS14 [Source:TAIR 3BAcc:55560] & 1.71 \\
\hline Atlg02790 & Exopolygalacturonase 4 (PGA4) [Source:Uniprot/SWISSPROT 3BAcc:P49062] & 1.67 \\
\hline
\end{tabular}




\begin{tabular}{|c|c|c|}
\hline At5g39880 & Transmembrane protein [Source:TAIR 3BAcc:39880] & 1.64 \\
\hline At5g44420 & $\begin{array}{l}\text { PLANT DEFENSIN 1.2A/Defensin-like protein } 16 \text { [Source:Uniprot/SWISSPROT } \\
\text { 3BAcc:Q9FI23] }\end{array}$ & 1.58 \\
\hline At5g07430 & $\begin{array}{l}\text { Pectin lyase-like superfamily protein/Probable } \\
\text { [Source:Uniprot/SWISSPROT 3BAcc:Q9LY17] }\end{array}$ & 1.57 \\
\hline At $\lg 07260$ & 71C3 (UGT71C3) [Source:Uniprot/SWISSPROT & 1.54 \\
\hline At2g26020 & $\begin{array}{l}\text { PLANT DEFENSIN } \quad \text { 1.2B/Putative } \\
\text { [Source:Uniprot/SWISSPROT 3BAcc:O80994] }\end{array}$ & 1.52 \\
\hline At5g19580 & Glyoxal oxidase-related protein [Source:TAIR 3BAcc:19580] & 1.51 \\
\hline At $2 g 26010$ & $\begin{array}{l}\text { PLANT DEFENSIN 1.3/Defensin-like protein } 14 \text { [Source:Uniprot/SWISSPROT } \\
\text { 3BAcc:O80995] }\end{array}$ & 1.47 \\
\hline $1+5 g 45880$ & Pollen Ole e 1 allergen and extensin family protein [Source:TAIR 3BAcc:45880] & 1.45 \\
\hline At5g50030 & $\begin{array}{l}\text { Plant invertase/pectin methylesterase inhibitor superfamily protein [Source:TAIR } \\
\text { 3BAcc:50030] }\end{array}$ & 1.42 \\
\hline t $3 g 01240$ & Splicing regulatory glutamine/lysine-rich-like protein & 1.35 \\
\hline t3g62710 & 1 hydrolase family protein [Source:T & 1.35 \\
\hline t5g04180 & Uniprot/SWISSPROT 3BAcc:Q9FYE3] & 1.33 \\
\hline At5g12960 & roline-tRNA ligase (DUF1680) [Source:TAIR 3BAcc:12960] & 1.33 \\
\hline At5g26700 & $\begin{array}{l}\text { germin-like protein subfamily } 2 \text { member } \\
\text { 2] }\end{array}$ & 1.26 \\
\hline Atlg05580 & $\begin{array}{l}\text { Cation/H(+) antiporter } 232 \mathrm{C} \text { chloroplastic (CHX23) [Source:Uniprot/SWISSPROT } \\
\text { 3BAcc:Q8VYD4] }\end{array}$ & 1.26 \\
\hline t5g58390 & Peroxidase superfamily protein [Source:Uniprot/SWISSPROT 3BAcc:Q9LVL2] & 1.23 \\
\hline $\operatorname{tlg} 61566$ & SWISSPROT 3BA & 1.19 \\
\hline Atlg62760 & ce:TAIR 3BAcc:AT1G62760] & 1.18 \\
\hline Atlg06160 & $\begin{array}{l}\text { ive transcription factor ERF094/Ethylene responsive factor } 59 \\
\text { SWISSPROT 3BAcc:Q9LND1] }\end{array}$ & 1.16 \\
\hline At $2 g 04460$ & able element gene [Source:TAIR 3BAcc:04460] & 1.16 \\
\hline At5g61160 & $\begin{array}{l}\text { Agmatine coumaroyltransferase/Anthocyanine } \\
\text { [Source:Uniprot/SWISSPROT 3BAcc:Q9FNP9] }\end{array}$ & 1.13 \\
\hline 470 & in 5 [Source:TAIR 3BAcc & 1.09 \\
\hline $\operatorname{tlg} 59950$ & ource:TAIR 3BAcc:59950] & 1.08 \\
\hline At $\lg 75830$ & 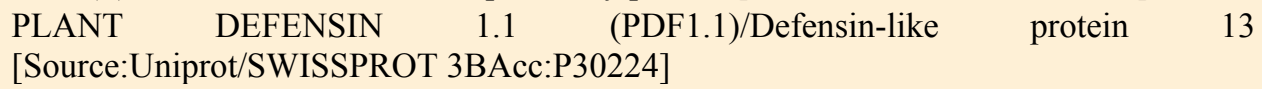 & 1.06 \\
\hline$A t 4 g$ & Putative beta-1,3-endoglucanase [Source:TAIR 3BAcc:16260] & 1.01 \\
\hline At2g41180 & $\begin{array}{l}\text { binding protein } 22 \mathrm{C} \text { chloroplastic (SIB2) [Source:Uniprot/SWISSPROT } \\
\text { 69] }\end{array}$ & 0.98 \\
\hline t3g49110 & PRX33) [Source. Uninret/SW & \\
\hline 90 & $\mathrm{C}$ & 0.95 \\
\hline 53 & 531 & 0.92 \\
\hline 80 & ] & 0.91 \\
\hline 63 & ISSPROT 3BAcc:Q1ECR9] & 0.90 \\
\hline 828 & 8] & 0.90 \\
\hline 515 & $\operatorname{Pr}$ & 0.88 \\
\hline At2g31141 & & 0.88 \\
\hline At2g28210 & A & 0.85 \\
\hline At3g13400 & $\mathrm{R} 3 \mathrm{~B}$ & 0.82 \\
\hline At $2 g 22320$ & & 0.82 \\
\hline Atlg66390 & Transcription factor MYB90 [Source:Uniprot/SWISSPROT 3BAcc:Q9ZTC3] & 0.81 \\
\hline \\
\hline & $\begin{array}{l}\text { it family pr } \\
850]\end{array}$ & -2 \\
\hline Atlg29920 & $\begin{array}{l}\text { Chlorophyll a/b binding protein } 2 \text { (LHCB1.2) }[\mathrm{So} \\
\text { 3BAcc:Q8VZ87] }\end{array}$ & -1.7 \\
\hline
\end{tabular}




\begin{tabular}{|c|c|}
\hline $2 g 34430$ & $\begin{array}{l}\text { Light-harvesting chlorophyll-protein complex } \text { II subunit B1 } \\
\text { 3BAcc:At2g34430] }\end{array}$ \\
\hline At3g27690 & Photosystem II light harvesting complex gene 2.3 [Source:TAIR 3BAcc:At3g27690] \\
\hline $4 t 2 g 05100$ & Photosystem II light harvesting complex gene 2.1 [Source:TAIR 3BAcc:At2g05100] \\
\hline $1 t \lg 29910$ & $\begin{array}{l}\text { Light-harvesting chlorophyll } \mathrm{a} / \mathrm{b} \text { binding protein } 1.2 \text { [Source:TAIR } \\
\text { 3BAcc:At1g29910] }\end{array}$ \\
\hline t2g05070 & Photosystem II light harvesting complex gene 2.2 [Source:TAIR 3BAcc:At2g05070] \\
\hline $\operatorname{t3g} 19390$ & Granulin repeat cysteine protease family protein [Source:TAIR 3BAcc:At3g19390] \\
\hline At3g19710 & BCAT4 [Source:Uniprot/SWISSPROT \\
\hline Atlg62510 & $\begin{array}{l}\text { Bifunctional inhibitor/lipid-transfer protein/seed storage } 2 \mathrm{~S} \text { albumin superfamily } \\
\text { protein [Source:TAIR 3BAcc:At1g62510] }\end{array}$ \\
\hline $4 g 21650$ & Subtilase 3. 13 (SBT3.13) [Source:TAIR 3BAcc:At4g21650] \\
\hline $5 g 65690$ & yruvate carboxykinase 2 (PCK2) [Source:TAIR 3BAcc:At5 \\
\hline $5 g 24490$ & 3BAcc:At5g24490] \\
\hline $4 t \lg 29930$ & $\begin{array}{l}\text { b binding protein } 12 \mathrm{C} \text { chloroplastic/Light-harvesting chlorophyll a/b } \\
\text { urce:Uniprot/SWISSPROT 3BAcc:P04778] }\end{array}$ \\
\hline 270 & g chlorophyll B-binding protein 3 [Source:TAIR 3BAcc:At5g54270] \\
\hline At5g38420 & $\begin{array}{l}\text { hosphate carboxylase small chain 2B 2C chloroplastic (R } \\
\text { ot/SWISSPROT 3BAcc:P10797] }\end{array}$ \\
\hline 80 & ein [Source:TA \\
\hline Atlg16410 & $\begin{array}{l}\text { Dihomomethionine N-hydroxylase/Cytochrome } \\
\text { [Source:Uniprot/SWISSPROT 3BAcc:Q949U1] }\end{array}$ \\
\hline At4g & oxygenase 4 (MIOX4) [Source:Uniprot/SWISSPROT 3BAcc:Q8H1S0] \\
\hline 60 & Q 2C chloroplastic [Source:Uniprot/SWISSPROT 3BAcc:Q9LU86] \\
\hline At $5 g C$ & racting protein [Source:TAIR 3BAcc:At5g02160] \\
\hline At $5 g 6$ & $\begin{array}{l}\text { Photosystem I reaction center subunit } \\
\text { [Source:Uniprot/SWISSPROT 3BAcc:P49107] }\end{array}$ \\
\hline At3g & $\begin{array}{l}\text { Homocysteine } \\
\text { 3BAcc:Q8LAX0] }\end{array}$ \\
\hline At2g10940 & $\begin{array}{l}\text { Bifunctional inhibitor/lipid-transfer protein/seed storage } 2 \mathrm{~S} \text { albumin superfamily } \\
\text { protein [Source:TAIR 3BAcc:AT2G10940] }\end{array}$ \\
\hline At3g & 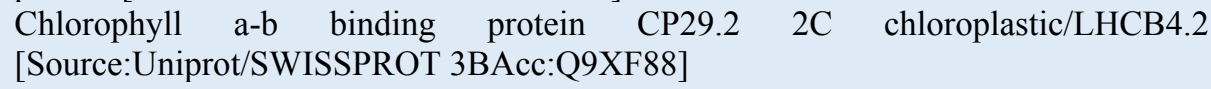 \\
\hline At4g01330 & nase superfamily protein [Source:TAIR 3BAcc:At4g01330] \\
\hline Atlg30280 & $\begin{array}{l}\text { Chaperone DnaJ-domain } \\
\text { 3BAcc:At1g30280] }\end{array}$ \\
\hline 570 & ar phosphate exchanger (DUF506) [Source:TAIR 3BAcc:At2g20670] \\
\hline At $2 g 3$ & $\begin{array}{l}\text { Photosystem II light harvesting complex gene B1B2 } \\
\text { 3BAcc:At2g34420] }\end{array}$ \\
\hline Atlg68560 & $\begin{array}{l}\text { Alpha-xylosidase } 11 \text { (ATXYL1)/Altered } \\
\text { [Source:Uniprot/SWISSPROT 3BAcc:Q9S7Y7] }\end{array}$ \\
\hline At4g08870 & $\begin{array}{l}\text { ne amidohydrolase } 2 \\
\text { :Q9ZPF5] }\end{array}$ \\
\hline At5g23010 & $\begin{array}{l}\text { Methylthioalkylmalate synthase } 12 \mathrm{C} \text { chloroplastic [Source:Uniprot/SWISSPROT } \\
\text { 3BAcc:Q9FG67] }\end{array}$ \\
\hline At3g50800 & \\
\hline At2g46740 & SPROT 3 \\
\hline At5g63450 & SPROT 3BA \\
\hline At4g03060 & kyl producing 2 (AOP2) [Source:TAIR 3BAcc:At4g03060] \\
\hline At5g55150 & $\begin{array}{l}\text { F-box SKIP23-like protein } \quad \text { (DUF295) } \\
\text { 3BAcc:Q9FLP7] }\end{array}$ \\
\hline At $\lg 12900$ & $\begin{array}{l}\text { Glyceraldehyde-3-phosphate dehydrogenase } \\
\text { [Source:Uniprot/SWISSPROT 3BAcc:Q9LPW0] }\end{array}$ \\
\hline At3 $\xi$ & rot/SWISSPROT 3BAcc:Q9S702] \\
\hline Atlg68010 & $\begin{array}{l}\text { Glycerate dehydrogenase HPR } 2 \mathrm{C} \text { peroxisomal [Source:Uniprot/SWISSPROT } \\
\text { 3BAcc:Q9C9W5] }\end{array}$ \\
\hline 80 & Plastid transcriptionally active 16 [Source:TAIR 3BAcc:AT3G46780] \\
\hline
\end{tabular}




\begin{tabular}{|c|c|}
\hline $4 t 5 g 14740$ & $\begin{array}{l}\text { Beta carbonic anhydrase } 2 \text { 2C chloroplastic [Source:Uniprot/SWISSPROT } \\
\text { 3BAcc:P42737] }\end{array}$ \\
\hline Atlg32060 & Phosphoribulokinase 2C chloroplastic [Source:Uniprot/SWISSPROT 3BAcc:P25697] \\
\hline At4g10340 & $\begin{array}{l}\text { Light-harvesting complex of Photosystem } \\
\text { [Source:Uniprot/SWISSPROT 3BAcc:Q9XF89] }\end{array}$ \\
\hline At4g28040 & $\begin{array}{l}\text { Nodulin MtN21-like transporter family } \\
\text { [Source:Uniprot/SWISSPROT 3BAcc:Q9SUD5] }\end{array}$ \\
\hline Atlg01620 & Aquaporin PIP1;3 [Source:Uniprot/SWISSPROT 3BAcc:Q08733] \\
\hline At5g. & $\begin{array}{l}\text { Probable xyloglucan endotransglucosylase/hydrolase } \\
\text { [Source:Uniprot/SWISSPROT 3BAcc:Q38907] }\end{array}$ \\
\hline At2g13360 & $\begin{array}{l}\text { L-Serine: glyoxylate aminotransferase (SGAT) [Source:Uniprot/SWISSPROT } \\
\text { 3BAcc:Q56YA5] }\end{array}$ \\
\hline At2g46820 & $\begin{array}{l}\text { Curvature thylakoid 1B 2C chloroplastic (CURT1B) [Source:Uniprot/SWISSPROT } \\
\text { 3BAcc:Q8LCA1] }\end{array}$ \\
\hline At5g13630 & $\begin{array}{l}\text { Magnesium-chelatase subunit ChlH 2C chloroplastic/ABA-binding protein (ABAR) } \\
\text { [Source:Uniprot/SWISSPROT 3BAcc:Q9FNB0] }\end{array}$ \\
\hline
\end{tabular}


Supplementary Table 3. The most strongly affected gene ontology (GO) terms assigned to upregulated or downregulated differentially expressed genes (adjusted $P$-value $<$ 0.05) in Arabidopsis leaves treated with 3-methoxy-BAPA for $6 \mathrm{~h}$

\begin{tabular}{|c|c|c|c|c|}
\hline GO number & Domain & Description & Total \# & Affected genes (\%) \\
\hline \multicolumn{5}{|l|}{ Up-regulated } \\
\hline GO:0009861 & $\mathrm{BP}$ & jasmonic acid and ethylene-dependent systemic resistance & 13 & 23.1 \\
\hline GO:0016653 & MF & oxidoreductase activity, acting on $\mathrm{NAD}(\mathrm{P}) \mathrm{H}$, heme protein as acceptor & 9 & 22.2 \\
\hline GO:0047893 & MF & flavonol 3-O-glucosyltransferase activity & 11 & 18.2 \\
\hline GO:0004089 & MF & carbonate dehydratase activity & 14 & 14.3 \\
\hline GO:0006026 & $\mathrm{BP}$ & aminoglycan catabolic process & 24 & 12.5 \\
\hline GO:0046348 & $\mathrm{BP}$ & amino sugar catabolic process & 24 & 12.5 \\
\hline GO:1901072 & $\mathrm{BP}$ & glucosamine-containing compound catabolic process & 24 & 12.5 \\
\hline GO:0006032 & $\mathrm{BP}$ & chitin catabolic process & 24 & 12.5 \\
\hline GO:0080043 & MF & quercetin 3-O-glucosyltransferase activity & 24 & 12.5 \\
\hline GO:0016998 & $\mathrm{BP}$ & cell wall macromolecule catabolic process & 27 & 11.1 \\
\hline GO:0071456 & $\mathrm{BP}$ & cellular response to hypoxia & 26 & 7.7 \\
\hline GO:0071804 & $\mathrm{BP}$ & cellular potassium ion transport & 43 & 7.0 \\
\hline GO:0009817 & BP & defense response to fungus, incompatible interaction & 44 & 6.8 \\
\hline GO:0004364 & MF & glutathione transferase activity & 54 & 5.6 \\
\hline GO:0019722 & $\mathrm{BP}$ & calcium-mediated signaling & 55 & 5.5 \\
\hline \multicolumn{5}{|c|}{ Down-regulated } \\
\hline GO:0030076 & $\mathrm{CC}$ & light-harvesting complex & 26 & 61.5 \\
\hline GO:0009522 & $\mathrm{CC}$ & photosystem I & 42 & 59.5 \\
\hline GO:0010196 & $\mathrm{BP}$ & nonphotochemical quenching & 7 & 57.1 \\
\hline GO:0019253 & $\mathrm{BP}$ & reductive pentose-phosphate cycle & 20 & 55.0 \\
\hline GO:0009765 & $\mathrm{BP}$ & photosynthesis, light harvesting & 39 & 53.9 \\
\hline GO:0016168 & MF & chlorophyll binding & 38 & 52.6 \\
\hline GO:0019685 & $\mathrm{BP}$ & photosynthesis, dark reaction & 21 & 52.4 \\
\hline GO:0015977 & $\mathrm{BP}$ & carbon fixation & 22 & 50.0 \\
\hline GO:0042549 & $\mathrm{BP}$ & photosystem II stabilization & 6 & 50.0 \\
\hline GO:0009533 & $\mathrm{CC}$ & chloroplast stromal thylakoid & 8 & 50.0 \\
\hline GO:0008187 & MF & poly-pyrimidine tract binding & 18 & 44.4 \\
\hline GO:0008266 & MF & poly(U) RNA binding & 18 & 44.4 \\
\hline GO:0009773 & $\mathrm{BP}$ & photosynthetic electron transport in photosystem I & 48 & 41.7 \\
\hline GO:0018298 & $\mathrm{BP}$ & protein-chromophore linkage & 41 & 41.5 \\
\hline
\end{tabular}


GO, gene ontology; BP, biological process; CC, cellular component; MF, molecular function.

Detached Arabidopsis leaves were treated with $10 \mu \mathrm{M} 3$-methoxy-BAPA or an equivalent volume of buffer containing $0.1 \%$ DMSO (negative control) for $6 \mathrm{~h}$ in darkness. The

most strongly affected GO terms at level 6 or higher are shown. Percentages of DEGs are calculated based on the total number of genes in Arabidopsis thaliana genome annotated with the same GO number (total \#). 
Supplementary Table 4. Effect of the treatment with 3-methoxy-BAPA (3MeOBAPA) and 3-hydroxy-BAPA (3OHBAPA) on the grain yield, number of tillers and number of spikes in winter wheat / spring barley plants. The same plants were used in field-plot experiments evaluating the level of plant infection by fungal pathogens (see Figure 7). The $5 \mu \mathrm{M}$ solutions were applied as a foliar spray at different plant growth stages (tillering, the beginning of stem elongation, the beginning of flag leaf extending). The values in bold italics show statistically significant difference between treated and non-treated plants $(P<0.01)$. n.d., not determined.

\begin{tabular}{|c|c|c|c|c|c|c|c|}
\hline & \multirow{2}{*}{$\begin{array}{c}\text { Compound } \\
\text { (growth stage } \\
\text { during application) }\end{array}$} & \multicolumn{2}{|c|}{ Grain yield } & \multicolumn{2}{|c|}{$\begin{array}{c}\text { Number of tillers } \\
\text { per plant }\end{array}$} & \multicolumn{2}{|c|}{$\begin{array}{c}\text { Number of spikes } \\
\text { per } \mathbf{m}^{2}\end{array}$} \\
\hline & & {$\left[\mathrm{t} \mathrm{ha}^{-1}\right]$} & {$[\%]$} & & [\%] & & {$[\%]$} \\
\hline \multirow[t]{2}{*}{ wheat } & non-treated & 6.72 & 100 & 5.9 & 100 & 432 & 100 \\
\hline & $\begin{array}{c}\text { 3MeOBAPA } \\
\text { (tillering) }\end{array}$ & 6.48 & 96 & 6.3 & 107 & 470 & 109 \\
\hline \multirow[t]{5}{*}{ barley } & non-treated & 8.53 & 100 & 3.8 & 100 & 698 & 100 \\
\hline & $\begin{array}{c}\text { 3MeOBAPA } \\
\text { (stem elongation) }\end{array}$ & 8.78 & 103 & 4.7 & 124 & 748 & 107 \\
\hline & $\begin{array}{c}\text { 3MeOBAPA } \\
\text { (flag leaf extending) }\end{array}$ & 8.67 & 102 & n.d. & n.d. & 721 & 103 \\
\hline & $\begin{array}{c}\text { 3OHBAPA } \\
\text { (stem elongation) }\end{array}$ & 8.62 & 101 & 4.2 & 111 & 688 & 99 \\
\hline & $\begin{array}{c}\text { 3OHBAPA } \\
\text { (flag leaf extending) }\end{array}$ & 8.86 & 104 & n.d. & n.d. & 686 & 98 \\
\hline
\end{tabular}

Supplementary Table 5. Primers for expression profiling of $A$. thaliana MAPK genes.

\begin{tabular}{|c|c|c|}
\hline Gene & AGI No. & Primers 5' - 3' \\
\hline \multicolumn{3}{|c|}{ Housekeeping genes (Endogenous controls) } \\
\hline Act2 & At3g18780 & $\begin{array}{l}\text { TGGTCGTACAACCGGTATTGTG } \\
\text { ATCAGTAAGGTCACGTCCAGCAA }\end{array}$ \\
\hline Act7 & At5g09810 & $\begin{array}{l}\text { CTAGAGACAGCCAAGAGCAGTTC } \\
\text { GTTTCATGGATTCCAGGAGCTTC }\end{array}$ \\
\hline $\mathrm{EF} 1 \alpha$ & At5g60390 & $\begin{array}{l}\text { TGAGCACGCTCTTCTTGCTTTCA } \\
\text { GGTGGTGGCATCCATCTTGTTACA }\end{array}$ \\
\hline \multicolumn{3}{|c|}{ MAPK/PTI marker genes } \\
\hline MPK3 & At3g45640 & $\begin{array}{l}\text { GACAGAGTTGCTTGGCACACCGA } \\
\text { GGCTGACGTGGGAAGTTGGGA }\end{array}$ \\
\hline MPK4 & At4g01370 & $\begin{array}{l}\text { TGTCGGCTGGTGCAGTCGATTT } \\
\text { TGGCACAACGCCTCATCAACTGT }\end{array}$ \\
\hline MPK6 & At2g43790 & $\begin{array}{l}\text { ACAGCTTCCACCTTATCCTCGCCA } \\
\text { TGGGCCAATGCGTCTAAAACTGTG }\end{array}$ \\
\hline MPK11 & At $\lg 01560$ & $\begin{array}{l}\text { TTCTTAAGAAGCGACAACGCTAG } \\
\text { ATTGACCGACATGTTTGGGAATC }\end{array}$ \\
\hline FRK1 & At2g19190 & $\begin{array}{l}\text { GAGACTATTTGGCAGGTAAAAGGT } \\
\text { AGGAGGCTTACAACCATTGTG }\end{array}$ \\
\hline WRKY33 & At2g38470 & $\begin{array}{l}\text { GGGAAACCCAAATCCAAGA } \\
\text { GTTTCCCTTCGTAGGTTGTGA }\end{array}$ \\
\hline WRKY53 & At4g23810 & $\begin{array}{l}\text { CGGAAGTCCGAGAAGTGAAG } \\
\text { TCTGACCACTTTGGTAACATCTTT }\end{array}$ \\
\hline
\end{tabular}




\section{Supplementary References}

1. Gucký, T., Jorda, R., Zatloukal, M., Bazgier, V., Berka, K., Řezníčková, E., Béres, T., Strnad, M., and Kryštof, V. (2013) A novel series of highly potent 2,6,9-trisubstituted purine cyclindependent kinase inhibitors, J. Med. Chem. 56, 6234-6247.

2. Kanehisa, M., Sato, Y., Kawashima, M., Furumichi, M., and Tanabe, M. (2016) KEGG as a reference resource for gene and protein annotation, Nucleic Acids Res. 44, D457-462.

3. Suzuki, T., Miwa, K., Ishikawa, K., Yamada, H., Aiba, H., and Mizuno, T. (2001) The Arabidopsis sensor His-kinase, AHK4, can respond to cytokinins, Plant Cell Physiol. 42, 107-113.

4. Yamada, H., Suzuki, T., Terada, K., Takei, K., Ishikawa, K., Miwa, K., Yamashino, T., and Mizuno, T. (2001) The Arabidopsis AHK4 histidine kinase is a cytokinin-binding receptor that transduces cytokinin signals across the membrane, Plant Cell Physiol. 42, 1017-1023.

5. Spíchal, L., Rakova, N. Y., Riefler, M., Mizuno, T., Romanov, G. A., Strnad, M., and Schmülling, T. (2004) Two cytokinin receptors of Arabidopsis thaliana, CRE1/AHK4 and AHK3, differ in their ligand specificity in a bacterial assay, Plant Cell Physiol. 45, 1299-1305.

6. Romanov, G. A., Spíchal, L., Lomin, S. N., Strnad, M., and Schmülling, T. (2005) A live cell hormone-binding assay on transgenic bacteria expressing a eukaryotic receptor protein. Anal. Biochem. 347, 129-134.

7. Meier, U. (2001). Growth stages of mono- and dicotyledonous plants. BBCH Monograph, Federal Biological Research Centre for Agriculture and Forestry, Bonn. 